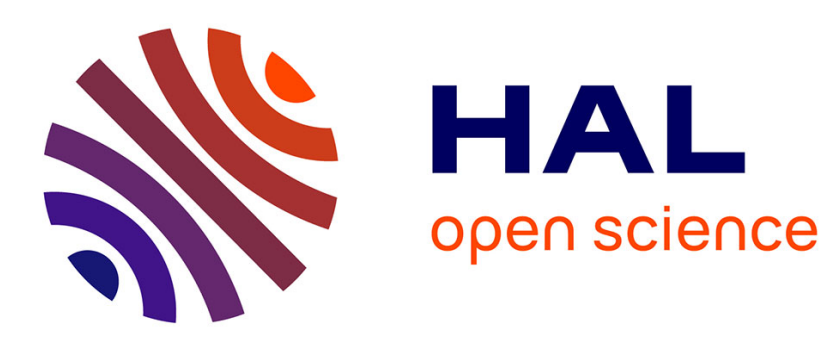

\title{
Détermination des marges de manoeuvre des élevages à partir de la mesure des inefficacités
}

\author{
I. Piot-Lepetit, P. Rainelli
}

\section{To cite this version:}

I. Piot-Lepetit, P. Rainelli. Détermination des marges de manoeuvre des élevages à partir de la mesure des inefficacités. INRA Productions Animales, 1996, 9 (5), pp.367-377. hal-01072891

\section{HAL Id: hal-01072891 \\ https://hal.science/hal-01072891}

Submitted on 1 Jun 2020

HAL is a multi-disciplinary open access archive for the deposit and dissemination of scientific research documents, whether they are published or not. The documents may come from teaching and research institutions in France or abroad, or from public or private research centers.
L'archive ouverte pluridisciplinaire HAL, est destinée au dépôt et à la diffusion de documents scientifiques de niveau recherche, publiés ou non, émanant des établissements d'enseignement et de recherche français ou étrangers, des laboratoires publics ou privés. 
INRA Prod. Anim., 1996, 9 (5),367-377

\section{PIOT-LEPETIT, P. RAINELLI}

INRA Unité d'Economie

et Sociologie rurales

65 rue de St-Brieuc 35042 Rennes Cedex
Détermination des marges de manœuvre des élevages à partir de la mesure des inefficacités

\section{On dit qu'il y a inefficacité technique lorsque le niveau de production qui est atteint pourrait l'être en utilisant moins d'intrants ou, autrement dit, en utilisant une meilleure combinaison des facteurs de production. La mesure de ces inefficacités permet de caractériser les améliorations qui pourraient être apportées.}

Traditionnellement, l'économie de la production se préoccupe de la façon dont les firmes choisissent leur plan de production. Les principes qui guident ce choix sont basés sur la maximisation du profit, sachant le prix

\section{Résumé}

Après une brève revue de la littérature économique sur la notion d'efficacité et sur son intérêt pour la gestion, cet article définit l'inefficacité technique comme la distance séparant tout niveau de production observé du niveau optimal qui serait obtenu si tous les intrants étaient utilisés de manière techniquement efficace. L'utilisation d'une méthodologie non paramétrique, appelée Data Envelopment Analysis (DEA), basée sur des techniques de programmation linéaire, permet la mesure de l'efficacité technique de trois échantillons du RICA (Réseau d'Information Comptable Agricole) pour l'année 1991 de producteurs de porcs ayant des orientations différentes : porcs seuls, porcs et herbivores ou granivores (porcs et volailles).

Les premiers résultats attribuent une plus grande efficacité moyenne aux producteurs de porcs. Ces derniers sont en général moins âgés et mieux formés que les producteurs des deux autres groupes. Ce résultat apparaît lié à la plus grande homogénéité des techniques de production mises en ouvre et à la prépondérance des facteurs variables par rapport aux facteurs fixes dans le processus de production. Pour les producteurs d'herbivores, les plus et les moins efficaces ont plutôt une orientation laitière. La différence de performance s'explique par une meilleure utilisation des consommations intermédiaires chez les plus efficaces. Enfin, le groupe de producteurs de granivores se rapproche de celui des éleveurs de porcs mais présente des caractéristiques moins marquées. Sur un plan environnemental, une compatibilité entre amélioration de l'efficacité technique et réduction des rejets azotés semble possible seulement chez les éleveurs d'herbivores et de granivores. Quant aux marges de manœuvre pour améliorer l'efficacité technique des producteurs, elles concernent à court terme principalement les charges liées à l'activité de production de végétaux et à plus long terme le facteur terre pour les exploitations hors sol et le matériel pour celles élevant des herbivores. Enfin, la mesure de l'efficacité d'échelle met en évidence des possibilités d'économies de coût suite à une modification de l'échelle de production pour plus de $52 \%$ des élevages quel que soit l'échantillon considéré. des produits et des facteurs de production, ou, ce qui revient au même, sur la minimisation du coût, connaissant le prix des intrants. Une telle démarche peut sembler assez théorique et, en fait, peu susceptible de guider pratiquement le bon fonctionnement des entreprises. A première vue, on est assez éloigné de la gestion qui " ne poursuit pas une connaissance désintéressée et contemplative, mais cherche à diriger les organismes de manière optimale ou, du moins, de la moins mauvaise manière possible " (Lassègue 1981). Pour cet auteur, la gestion ne relève pas «d'une connaissance gratuite, mais d'une connaissance tournée vers l'action ».

Sans entrer dans le débat plus général des rapports entre économie de l'entreprise et gestion (cf Glais 1990), nous voudrions montrer ici qu'un certain nombre de développements récents en économie de la production ouvrent la voie d'une réconciliation entre ce qui peut apparaître comme la " connaissance gratuite ", et la " connaissance tournée vers l'action ". Une telle réconciliation passe par l'établissement de mesures d'efficacité qui permettent l'évaluation des performances relatives dans un groupe d'unités productives.

L'application de ces méthodes à l'agriculture paraît particulièrement judicieuse dans la mesure où c'est un champ d'application privilégié de l'économie de la production, en raison des rapports relativement étroits entre économistes agricoles et utilisateurs, comme le note Mairesse (1993). De plus l'économie agricole est, en amont, à l'origine des principales innovations intervenues en économie de la production (Chambers 1988). 
On se trouve donc à l'intersection des besoins des praticiens, pressés par les nouvelles règles du jeu résultant du mouvement général de libéralisation du secteur, et d'un certain nombre d'avancées théoriques.

L'article est structuré autour de deux axes : le premier à caractère plutôt méthodologique, le second étant basé sur l'analyse concrète de résultats. La première partie est consacrée à la notion d'inefficacité et à la présentation d'une méthode particulière de calcul, en s'aidant de graphiques simplifiés. La deuxième partie met en œuvre la méthode évoquée en proposant des estimations des inefficacités pour trois groupes d'exploitations d'élevage produisant des porcins mais ayant des orientations différentes (porcs seuls, porcs et herbivores, porcs et volailles). Il s'agit d'échantillons issus du Réseau d'Information Comptable Agricole (RICA) pour l'année 1991. Ces résultats permettent d'illustrer l'intérêt de l'approche proposée.

\section{1 / La notion d'inefficacité et son estimation par l'approche non paramétrique}

La notion d'inefficacité est précisée par référence à son contraire, l'efficacité, telle qu'elle est entendue dans la théorie économique depuis Pareto, et différents types d'inefficacité sont définies.

\section{1 / La notion d'inefficacité et son intérêt pour la gestion}

Dans le système de concurrence élaboré par les économistes, le marché joue un rôle fondamental dans l'allocation optimale des ressources entre les entrepreneurs, en recherchant l'efficacité sociale (ou collective). Cette efficacité correspond à une situation où il n'est plus possible de dégager de surplus distribuable sans pénaliser un individu. Ce mécanisme repose toutefois sur la présence d'agents rationnels et efficaces, c'est-à-dire qui produisent un niveau maximum de produits compte tenu de la technologie et des marchés des produits et des facteurs.

Si l'on a beaucoup discuté sur le rôle de la concurrence pure et parfaite par rapport au fonctionnement des marchés, on s'est beaucoup moins préoccupé de l'efficacité des producteurs et des conséquences qui pouvaient en résulter tant au niveau collectif qu'au niveau des performances des entreprises. Or les écarts entre le niveau maximum de production que l'on peut obtenir en intégrant toutes les contraintes auxquelles font face les producteurs, et la réalité sont sensibles et montrent l'existence d'importantes marges de manœuvre.

Sans réduire l'entreprise à une simple fonction de production, on peut quand même admettre, dans le cas de l'agriculture, que nous sommes en présence d'unités de faible dimension qui, prises individuellement, ne risquent pas d'influencer les conditions d'équilibre du marché. Ces unités subissent intégralement le système des prix, que celui-ci soit administré, comme pour le lait, ou plus ou moins libre, comme pour le porc. L'exploitant a tout juste la possibilité de fixer le volume de sa production à l'intérieur d'une certaine marge, qui peut plus ou moins varier selon les contraintes institutionnelles, avec l'accès au foncier, réglementaires, avec la limitation des effluents d'origine animale, ou administratives avec les droits à produire.

C'est à l'intérieur de cet ensemble de contraintes que le producteur va gérer son exploitation. Qu'il s'agisse de maximiser le profit, de minimiser le coût, ou de chercher à produire le plus possible compte tenu des facteurs de production disponibles et de leur prix, la notion de fonction frontière est utilisée dans chaque situation. La distance existant entre les exploitations et cette frontière donne une mesure de leur inefficacité, les plus efficaces étant évidemment situées sur celle-ci.

La mise en évidence de ces inefficacités, et surtout de leur origine, donne des indications sur les possibilités d'amélioration des résultats économiques, soit en produisant plus avec le même niveau de facteurs, soit en produisant autant avec moins d'intrants. C'est à Debreu (1951) que l'on doit la première mesure de l'efficacité avec le " coefficient d'utilisation des ressources " calculé à partir de la réduction équiproportionnelle maximale de tous les intrants permettant la production continue des produits fabriqués. Un tel coefficient donne une évaluation numérique de la perte associée à une situation non optimale. Toutefois, c'est Farrell (1957) qui définit de manière plus précise l'efficacité en dissociant ce qui est d'origine technique de ce qui est dû à un mauvais choix par rapport au prix des intrants.

L'efficacité technique, proche de l'esprit du coefficient d'utilisation des ressources de Debreu, mesure la façon dont l'entrepreneur combine les facteurs de production lorsque leurs proportions d'utilisation sont données. Il y a inefficacité technique quand on pourrait obtenir le même résultat avec une moindre quantité d'intrants. L'efficacité-prix se définit par la façon dont l'entrepreneur fixe les proportions entre les différents intrants participant à la combinaison productive en se basant sur leurs prix respectifs. Cette mesure donne une appréciation de la manière dont les firmes allouent leurs ressources productives par rapport à un objectif de production. D'où l'appellation d'efficacité allocative souvent utilisée en lieu et place du terme d'efficacitéprix employé par Farrell. La figure 1 donne une représentation graphique de ces notions.

Considérons une exploitation A qui utilise deux facteurs de production $\left(\mathrm{x}_{1}, \mathrm{x}_{2}\right)$ disponibles à un certain niveau de prix fixé pour produire un produit $\mathrm{y}$. La frontière de production décrite par yy' exprime le montant maxi- 
Figure 1. Mesures de l'inefficacité technique et allocative dans le plan $x_{1} x_{2}$ des facteurs de production.

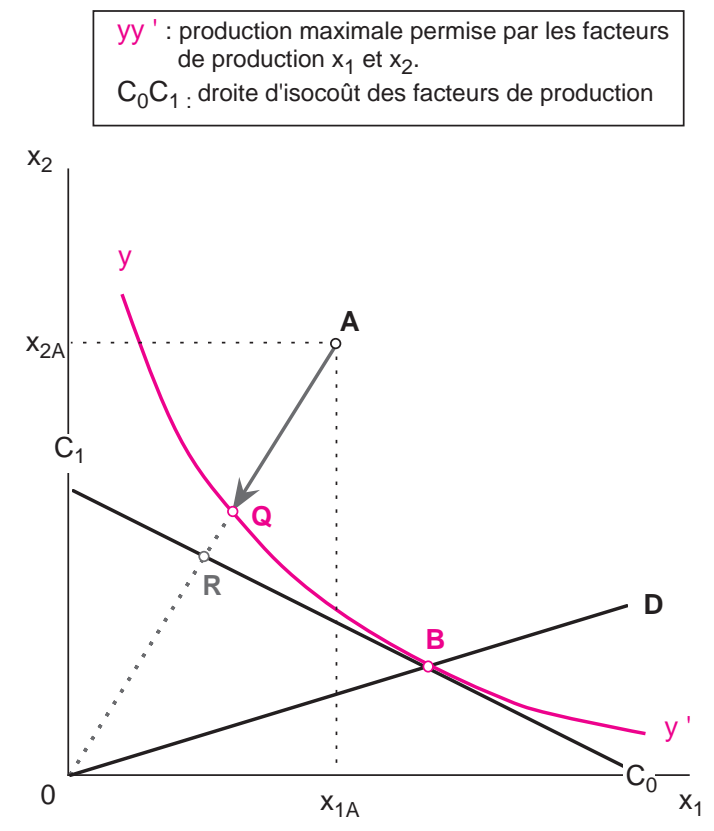

mum de produit pouvant être obtenu à partir de toute combinaison des deux intrants. En A, l'exploitation présente une inefficacité technique car elle se situe au-dessus de la frontière pour un niveau donné de produit. En réduisant l'utilisation de ses deux facteurs de production $\mathrm{x}_{1}$ et $\mathrm{x}_{2}$ le long du rayon OA jusqu'en $\mathrm{Q}$, l'exploitation peut produire le même niveau de bien à partir d'un vecteur plus petit d'intrants sans modifier la combinaison des facteurs utilisés. Avec cette réduction $\mathrm{OQ} / \mathrm{OA}$, l'exploitation A réalise une économie sur les coûts de production mis en œuvre sans perte de produit. Ainsi la mesure OQ/OA décrit l'efficacité technique de A. Numériquement elle prend des valeurs comprises entre 0 et 1 , avec un score d'efficacité technique de 1 pour toute exploitation située sur la frontière de production.

Si à présent on suppose que l'on est en présence d'un marché des facteurs de production concurrentiel, les prix relatifs des deux intrants $x_{1}$ et $x_{2}$ peuvent être représentés par la droite d'isocoût $\mathrm{C}_{0} \mathrm{C}_{1}$. Le point de contact $\mathrm{B}$ entre cette droite d'isocoût, et la frontière de production yy', donne le coût minimum de production pour la fabrication d'une unité de produit. Le point $R$, sur la droite d'isocoût, a le même coût que le point $B$. Le point $Q$, qui est sur la frontière dans le prolongement de OR, correspond à un emploi de ressources productives plus important. La distance RQ mesure le surcoût d'utilisation des facteurs $\mathrm{x}_{1}$ et $\mathrm{x}_{2}$. Compte tenu du rapport des prix existants, nous n'avons pas la combinaison de facteurs optimale. RQ représente donc l'inefficacité-prix ou inefficacité allocative de l'exploitation A.

Globalement l'exploitation A cumule une double inefficacité, technique et allocative que l'on peut exprimer par le rapport OR/OA. Celui-ci peut s'écrire :

$$
\mathrm{OR} / \mathrm{OA}=\mathrm{OQ} / \mathrm{OA} \times \mathrm{OR} / \mathrm{OQ}
$$

c'est-à-dire que l'inefficacité totale est le produit de l'inefficacité technique par l'inefficacité-prix. Dans le même ordre d'idée, l'exploitation D présente une inefficacité technique tout en étant allocativement efficace. Seule B est techniquement et allocativement efficace.

Indépendamment des inefficacités techniques et allocatives, une autre forme d'inefficacité peut se manifester, liée à l'écart existant entre les performances constatées et celles qui seraient obtenues dans une situation d'équilibre concurrentiel de long terme. Cet écart résulte de la présence d'économies d'échelle dans la situation observée, d'où l'existence d'une inefficacité d'échelle. Cet état des choses peut s'expliciter en partant de la fonction liant le coût de production aux quantités produites, connaissant le prix des intrants.

Cette fonction de coût $\mathrm{C}$ dépend de y (quantités produites) et de $\mathrm{p}$ (prix des intrants) soit $\mathrm{C}=\mathrm{C}(\mathrm{p}, \mathrm{y})$. Les économies d'échelle $\mathrm{E}(\mathrm{p}, \mathrm{y})$ vont s'apprécier à travers le rapport entre coût moyen $\mathrm{CM}$ et coût marginal $\mathrm{Cm}$. Elles sont croissantes quand $\mathrm{E}(\mathrm{p}, \mathrm{y})$ est supérieur à 1 , constantes quand $\mathrm{E}(\mathrm{p}, \mathrm{y})=1$ et décroissantes quand $\mathrm{E}(\mathrm{p}, \mathrm{y})$ est inférieur à 1 . Cette mesure équivaut aux rendements d'échelle obtenus en raisonnant à partir de la technologie, c'est-à-dire quand on examine la valeur du coefficient $\mathrm{k}$ ' par lequel est multiplié la production lorsqu'on multiplie les quantités d'intrants par $\mathrm{k}$.

Dans le cas particulier où le coût moyen est minimum, coût moyen et coût marginal s'égalisent et $\mathrm{E}(\mathrm{p}, \mathrm{y})=1$. On atteint alors un équilibre concurrentiel de long terme, avec un profit nul, où les facteurs quasi-fixes sont parfaitement adaptés au niveau de production. Il n'y a aucune sous-capacité d'emploi de la terre ou du travail familial et le prix du produit est égal au coût marginal. La mise en évidence d'un décalage entre coût moyen et coût marginal, par le biais des économies d'échelle permet donc la mise à jour d'une inefficacité d'échelle.

\section{2 / La mesure des inefficacités par l'approche non paramétrique}

Dans un deuxième point nous montrons comment, à partir d'une approche non paramétrique basée sur la programmation linéaire, un score d'efficacité par exploitation peut être calculé. L’approche développée selon les propositions initiales de Farrell (1957) est qualifiée de non paramétrique car on construit par programmation mathématique une enveloppe des observations sans qu'aucun vecteur de paramètres ne soit estimé. Charnes et al (1978) ont généralisé et rendu opérationnelles les propositions de Farrell en permettant l'estimation de la frontière à par- 
tir d'une courbe enveloppe formée de segments de droite reliant les unités efficaces, d'où le nom de Data Envelopment Analysis (DEA). La DEA fait reposer une surface linéaire par morceaux au sommet des observations et met en place un processus d'optimisation considérant chaque entreprise de manière individuelle.

La première étape de l'approche DEA est la construction d'une représentation de la technologie à partir de l'ensemble des observations dont on dispose. Il convient de noter le caractère relatif de la technologie définie ici puisque l'on raisonne à un moment donné en définissant une frontière de production à partir de l'échantillon d'exploitations considéré. Il est évident que hors de cet échantillon peuvent exister d'autres unités productives plus performantes. Ces unités performantes peuvent même être encore assez éloignées d'un optimum théorique qui peut de plus évoluer dans le temps par l'intégration de nouvelles techniques. Ce caractère statique et relatif de l'analyse doit être présent à l'esprit, notamment lors de l'interprétation des résultats.

La seconde étape de la DEA consiste à mesurer l'écart existant entre chaque exploitation observée et la frontière de production définie précédemment. Les valeurs des écarts sont comprises entre 0 et 1 . Toute exploitation observée située sur la frontière technologique définie en première étape se voit attribuer la valeur de 1 et est déclarée techniquement efficace, au moment considéré et relativement à l'échantillon. Les autres observations, situées à l'intérieur de l'ensemble de production ont un score inférieur à 1 et sont techniquement inefficaces par rapport aux exploitations ayant un score de 1 .

L'encadré page ci-contre présente la méthode de manière plus rigoureuse.

\section{2 / Inefficacités dans les élevages hors-sol et bovins}

La méthodologie DEA a été appliquée, à titre illustratif, à trois échantillons du RICA (Réseau d'Information Comptable Agricole) pour l'année 1991. Après une présentation des exploitations et la définition des variables utilisées pour décrire la technologie de production mise en œuvre, les résultats obtenus à partir des mesures d'efficacité technique et d'échelle sont présentés et commentés.

\section{1 / Caractéristiques des exploitations analysées}

L'étude s'est intéressée aux exploitations du RICA produisant des porcins pour l'année 1991. Celles-ci sont regroupées en 3 souséchantillons. Le premier nommé « Porcs seuls » contient 107 élevages de l'OTEX 50 (spécialisés en granivores) produisant, en moyenne, $97 \%$ de porcins. Le deuxième groupe dénommé " Herbivores " regroupe
129 exploitations de l'OTEX 71 (polyélevages à orientation herbivores). Les élevages concernés produisent, en moyenne, $70 \%$ de bovins et $25 \%$ de porcins. Enfin, le groupe "Granivores " contient des exploitations de l'OTEX 72 (polyélevages à orientation granivores) produisant $55 \%$ de porcs, $24 \%$ de volailles et $16 \%$ de bovins, en moyenne. Dans l'ensemble ces trois groupes produisent peu de végétaux : $5 \%$ de la production totale pour les porcs seuls et les granivores et $13 \%$ pour les herbivores.

Le groupe porcs seuls se situe à $77 \%$ en Bretagne avec une superficie moyenne par exploitation de 28 hectares de SAU. L'âge moyen des producteurs est de 41 ans et $60 \%$ d'entre eux ont une formation agricole secondaire ou supérieure. Les chefs d'exploitation de cette catégorie sont en moyenne plus jeunes et relativement plus formés que ceux des deux autres groupes. L'échantillon herbivores est beaucoup plus dispersé sur le territoire national, et ses exploitations ont une superficie moyenne de 48 hectares de SAU, la plus élevée des trois groupes considérés. Les chefs d'exploitation sont en moyenne plus âgés (45 ans) et moins formés que dans les deux autres échantillons. Seuls $29 \%$ d'entre eux ont un niveau de formation agricole secondaire ou supérieur. Le groupe granivores a une situation intermédiaire. Principalement situées en Bretagne, les exploitations de ce groupe ont une superficie moyenne de 39 hectares de SAU. L'âge moyen des chefs d'exploitation est de 44 ans avec un niveau de formation agricole secondaire ou supérieur pour 44 $\%$ d'entre eux. Quel que soit le groupe, il convient de noter que le travail est essentiellement familial avec une moyenne de 1,79 $\mathrm{UTA}^{(1)}$ pour les porcs seuls, 1,77 UTA pour les herbivores et 1,98 UTA pour les granivores.

\section{2 / Représentation de la technologie agricole}

Pour construire l'enveloppe technologique servant de référence aux mesures d'efficacité technique, il est nécessaire de définir les produits et les facteurs mis en œuvre dans la technologie de production. Pour les trois échantillons, trois produits sont pris en compte : la production animale, la production végétale et l'azote organique disponible, évalué à partir des effectifs des animaux présents sur l'exploitation et en se basant sur les normes du CORPEN ${ }^{(2)}$. Les deux premiers

(1) Une UTA (Unité de Travailleur Annuelle) est équivalente à 2200 heures de travail par an à raison de 8 heures par jour pendant l'exercice, soit 275 jours de 8 h. Pour un temps partiel, on calcule une UTA partielle égale au temps de travail effectué divisé par 2200 heures.

(2) L'évolution des excédents azotés est faite à partir d'un bilan simplifié des entrées et sorties proposé par le CORPEN (Comité d'Orientation pour la Réduction de la Pollution des Eaux par les Nitrates et les phosphates provenant des activités agricoles). 


\section{Approche DEA (Data Envelopment Analysis) et mesure de l'efficacité des exploitations}

\section{Première étape}

La DEA construit une représentation de la technologie à partir d'un ensemble d'observations. Pour ce faire, chaque exploitation est comparée aux autres entreprises dont elle-même. Si aucune observation ne produit plus avec la même dotation factorielle, ou autant avec une quantité d'intrants plus faible, alors l'exploitation appartient à la frontière de production, sinon elle est incluse dans l'ensemble des possibilités de production. Une enveloppe linéaire par morceaux est obtenue (cf. figure ci-dessous), sous hypothèse de convexité de la technologie, qui s'écrit, en termes analytiques comme suit :

$T=\left\{(x, y): y \leq \lambda Y, x \geq \lambda X, \sum_{j=1}^{J} \lambda_{j}=I, \lambda_{j} \geq 0, j=1 \ldots J\right\}$

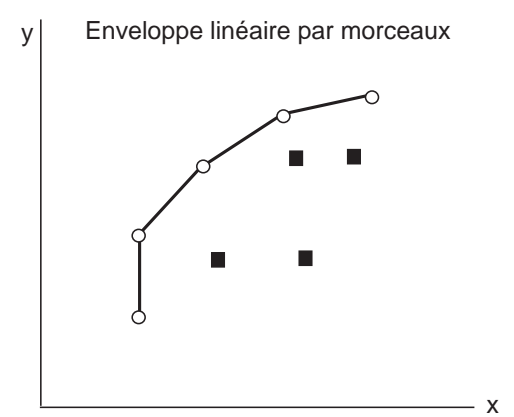

avec $y$ un vecteur de produits et $x$ un vecteur de facteurs de production comparés aux produits et facteurs observés contenus respectivement dans les matrices $Y$ de dimension $(J, M)$ et $X$ de dimension $(\mathrm{J}, \mathrm{N})$. La contrainte $\mathrm{y} \leq \lambda Y$ traduit le fait que l'on peut trouver une combinaison convexe de produits observés au moins aussi grande que le vecteur considéré et $x \geq \lambda X$ signifie qu'il existe une combinaison convexe de facteurs observés au plus aussi grande que le vecteur d'intrants considéré.

Cette enveloppe technologique $T$ fournit ainsi l'ensemble de tous les $\mathrm{x}$ capables de produire $\mathrm{y}$ et va servir de référence dans une deuxième étape pour mesurer l'efficacité des exploitations.

\section{Deuxième étape}

Lorsque l'on considère l'ensemble des facteurs de production permettant la production d'un niveau donné de produits, l'efficacité technique de Farrell

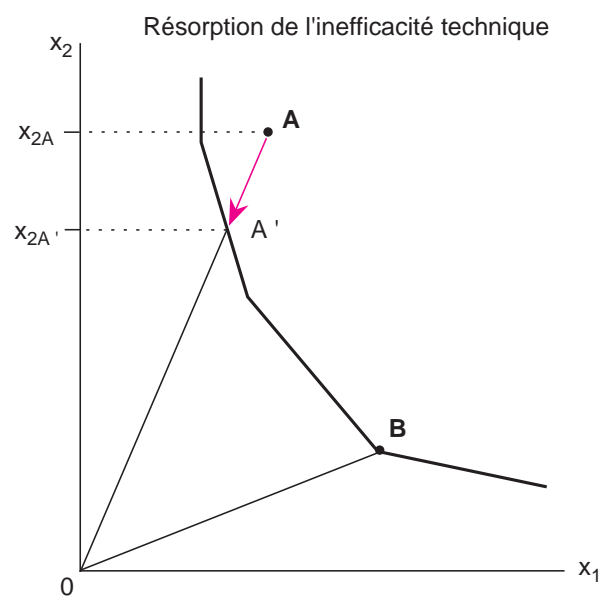

mesure la distance séparant toute observation de la frontière des facteurs permettant d'atteindre ce niveau fixé de production.

Comme l'illustre la figure ci-dessus, le point $A$ est techniquement inefficace car il ne se situe pas sur la frontière de l'ensemble des possibilités de facteurs. En A', projection de A le long du rayon OA, l'exploitation $A$ peut produire le même niveau de produits en consommant seulement $x_{2 A^{\prime}}$ de biens au lieu des $x_{2 A}$ observés, étant donné la technologie mise en œuvre dans l'échantillon considéré. Quant à l'observation B, elle est techniquement efficace relativement à l'échantillon car elle se situe sur la frontière de production.

La mesure de l'efficacité technique dans l'espace des facteurs d'une exploitation se définit par :

ET $(x, y)=\min \{h:(h x, y) \in T\} 0<h \leq 1$

avec $h$ la valeur du score d'efficacité technique.

Comme noté précédemment, l'écart ainsi mesuré peut aussi traduire une inefficacité liée à une échelle de production plus petite que celle d'autres exploitations de l'échantillon, du fait de la présence d'économies d'échelle présentes à court terme et pouvant être exploitées dans un plus long terme.

L'approche DEA permet de comparer plusieurs mesures d'efficacité technique obtenues sous différentes hypothèses de rendements d'échelle pour la représentation de la technologie définie dans la première étape. On peut ainsi définir une technologie à rendements d'échelle constants (ABOx sur la figure ci-dessous), à rendements d'échelle non croissants (ABDD'x) et à rendements d'échelle variable (C'CBDD'x).

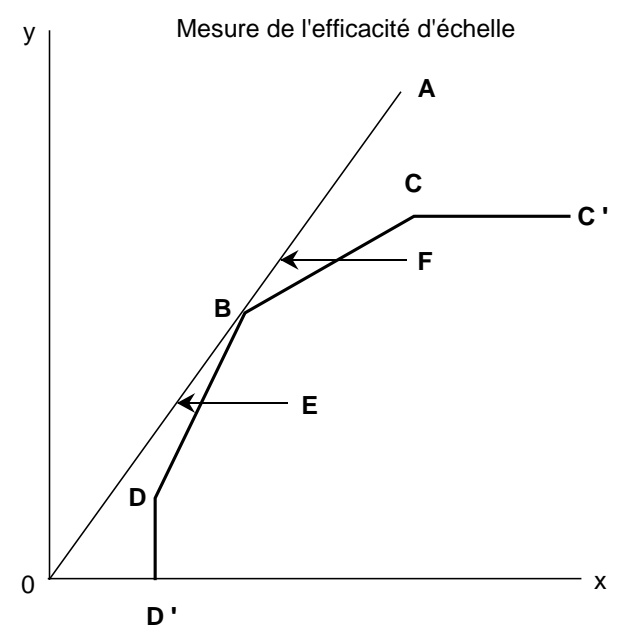

Comme l'illustre cette figure, la mesure de l'efficacité technique obtenue pour l'exploitation $\mathrm{E}$ par rapport à la technologie à rendements variables est plus faible que celles obtenues par rapport à la technologie à rendements constants ou non croissants. Ainsi, on en déduit que l'observation $\mathrm{E}$ présente, à court terme, des rendements d'échelle croissants et donc que des économies de coût peuvent être obtenues pour cette exploitation en augmentant son niveau de production. Inversement pour $F$, qui présente des rendements d'échelle décroissants, c'est en réduisant le volume produit que les économies peuvent apparaître.

\section{La méthode consiste}

à construire une "frontière de production", qui représente la production maximale pouvant être obtenue à partir des facteurs de production, puis à mesurer l'écart entre chaque exploitation et cette frontière : cet écart définit l'inefficacité technique. 
produits sont exprimés en francs et le dernier en kilogrammes.

Parmi les huit facteurs de production utilisés pour décrire la technologie, quatre d'entre eux sont supposés fixes car l'analyse ne concerne que l'année 1991. Ce sont : la terre (mesurée en hectares), le travail (mesuré en UTA) et le matériel et les bâtiments (exprimés en francs). Dans la variable matériel sont regroupées les charges de l'exercice liées à la location, à l'amortissement ou à l'entretien du matériel et dans la variable bâtiments sont pris en compte l'entretien des bâtiments ainsi que l'amortissement des constructions et installations complexes spécialisées.

Les quatre autres facteurs sont supposés variables ; ils correspondent à : l'azote minéral (exprimé en kg), les autres achats courants pour la production végétale, les charges pour la production animale ainsi que les autres charges d'exploitation. Ces trois dernières variables sont exprimées en francs. Le volume d'azote minéral utilisé sur chaque exploitation est déterminé à partir des achats d'engrais ${ }^{(3)}$. Le tableau 1 présente les valeurs moyennes des variables utilisées pour les différents échantillons.

Les charges d'élevage constituent un poste important des dépenses des exploitations. Elles représentent $77 \%$ des charges des producteurs du groupe porcs seuls, $67 \%$ de celles des granivores et $41 \%$ de celles des herbivores. Dans chaque groupe, l'achat d'aliments constitue plus de $75 \%$ de la dépense, atteignant même $92 \%$ de celle-ci pour l'échantillon porcs seuls. Les autres frais engagés sont les achats de combustibles, les frais de vétérinaires ou les travaux et services effectués par des tiers. Les charges affectées aux cultures correspondent principalement aux travaux et services effectués par des tiers pour l'activité de culture ainsi qu'à l'achat de produits phytosanitaires.

Notons que l'approche DEA n'utilise que des variables exprimées en volume. Cependant la nécessité d'agréger certaines variables nous conduit à les exprimer en valeur. Cette agrégation peut alors introduire un biais dans la mesure de l'efficacité technique car elle peut capturer l'effet du système des prix en plus des différences techniques existant entre les différentes exploitations. Toutefois, les tests mis en œuvre révèlent que ce biais est peu significatif (Piot-Lepetit 1995).

\section{3 / Mesure de l'efficacité technique}

L'efficacité technique des trois échantillons est présentée dans le tableau 2 en distinguant les agriculteurs performants avec un score unitaire, ceux qui le sont moins, avec un score compris entre 0,85 et 1 , et ceux dont le score est inférieur à 0,85 qui ne le sont pas. Si, par définition, le score moyen des meilleurs est identique dans les trois échantillons, on constate qu'il est assez semblable dans la classe intermédiaire (entre 0,93 pour le groupe porcs seuls et 0,98 pour celui des herbivores) tandis que les élevages les moins performants présentent une forte variabilité selon l'orientation. Ainsi le score d'efficacité technique atteint seulement 0,48 pour l'orientation herbivores, 0,64 pour les producteurs de granivores et 0,78 pour les éleveurs de porcs seuls.

En ce qui concerne la distribution des scores d'efficacité technique à l'intérieur des orientations étudiées, on constate que, quel que soit l'échantillon, plus de la moitié des exploitations ont un score égal à 1 . C'est dans le groupe herbivores que cette proportion est la plus élevée $(59 \%)$. Toutefois, la part rela-

(3) Le RICA distingue les charges d'engrais destinés aux cultures fourragères des charges d'engrais destinés aux autres types de culture. On a supposé que pour les cultures fourragères, les producteurs utilisent un ammonitrate 33,5 tandis que pour les cultures ils prennent un triple 17. Connaissant le prix moyen de l'unité d'azote de ces deux types d'engrais, on en déduit la quantité d'azote minéral utilisée.

Tableau 1. Valeurs des variables du modèle.

\begin{tabular}{|l|r|r|r|r|r|r|}
\hline & \multicolumn{2}{|c|}{ Porcs seuls } & \multicolumn{2}{c|}{ Herbivores } & \multicolumn{2}{c|}{ Granivores } \\
\cline { 2 - 7 } & moyenne & écart-type & moyenne & écart-type & moyenne & écart-type \\
\hline Produits & & & & & & \\
Produit animal (F) & 1628439 & 1189820 & 240491 & 238144 & 758250 & 595117 \\
Produit végétal (F) & 87946 & 108134 & 70274 & 74776 & 65573 & 110086 \\
Azote organique (kg) & 6480 & 4479 & 3778 & 2992 & 5863 & 3648 \\
Facteurs fixes & & & & & & \\
Terre (ha) & 28,20 & 16,87 & 47,70 & 27,71 & 39,01 & 21,30 \\
Travail (UTA) & 1,82 & 0,67 & 1,77 & 0,67 & 1,98 & 0,80 \\
Matériel (F) & 93406 & 77730 & 70218 & 53336 & 83997 & 55502 \\
Bâtiments (F) & 98263 & 80125 & 26156 & 30045 & 62924 & 50801 \\
Facteurs variables & & & & & & \\
Charges élevage (F) & 923652 & 673254 & 159408 & 216301 & 519804 & 451931 \\
Azote minéral (kg) & 3744 & 3062 & 8396 & 5822 & 5939 & 4467 \\
Charges cultures (F) & 68349 & 53713 & 51317 & 44981 & 55503 & 46258 \\
Autres charges (F) & 198483 & 268384 & 83442 & 77788 & 124997 & 90793 \\
\hline
\end{tabular}


Tableau 2. Scores d'efficacité technique.

\begin{tabular}{|c|c|c|c|c|c|c|c|c|c|}
\hline & \multicolumn{3}{|c|}{ Porcs seuls } & \multicolumn{3}{|c|}{ Herbivores } & \multicolumn{3}{|c|}{ Granivores } \\
\hline & Nombre & $\%$ & $\begin{array}{l}\text { Score } \\
\text { moyen }\end{array}$ & Nombre & $\%$ & $\begin{array}{l}\text { Score } \\
\text { moyen }\end{array}$ & Nombre & $\%$ & $\begin{array}{l}\text { Score } \\
\text { moyen }\end{array}$ \\
\hline $\begin{array}{l}\text { Efficacité technique } \\
\text { égale à } 1\end{array}$ & 55 & 51.4 & 1 & 76 & 58.9 & 1 & 71 & 52.6 & 1 \\
\hline entre 0,85 et 1 & 34 & 31,7 & 0,93 & 13 & 10,1 & 0,98 & 17 & 12,6 & 0,94 \\
\hline inférieur à 0,85 & 18 & 16,8 & 0,78 & 40 & 31,0 & 0,48 & 47 & 34,8 & 0,64 \\
\hline $\begin{array}{l}\text { Score moyen } \\
\text { (écart-type) } \\
\text { minimum }\end{array}$ & \multicolumn{3}{|c|}{$\begin{array}{c}0,94 \\
(0,085) \\
0,67\end{array}$} & \multicolumn{3}{|c|}{$\begin{array}{c}0,84 \\
(0,254) \\
0,19\end{array}$} & \multicolumn{3}{|c|}{$\begin{array}{c}0,87 \\
(0,191) \\
0,16\end{array}$} \\
\hline
\end{tabular}

tive des unités dont l'efficacité est comprise entre 0,85 et 1 diffère très nettement selon les types d'élevage avec une proportion proche de $32 \%$ pour les porcs et seulement 10 à $13 \%$ pour les herbivores et granivores. Logiquement, dans ces deux derniers échantillons, un tiers environ des exploitations ont une faible efficacité (score inférieur à 0,85 ). Il en résulte un score moyen très élevé pour les porcs seuls, avec 0,94 , tandis que pour les herbivores le score est le plus faible $(0,84)$, l'échantillon granivores étant intermédiaire $(0,87)$.

Les résultats précédents suggèrent plusieurs réflexions. En premier lieu, on peut attribuer la supériorité moyenne de l'efficacité technique chez les producteurs de porcs à l'existence pour eux d'une technologie standard recourant essentiellement aux facteurs variables, les aliments concentrés en l'occurrence. Cette prépondérance des facteurs variables par rapport aux facteurs fixes signifie une meilleure saturation de ces derniers. Cela est vrai pour le travail familial qui peut faire l'objet d'un emploi régulier dans l'année, et plus encore pour la terre. Rappelons que la taille moyenne des exploitations productrices de porcs est de 28 ha contre 48 pour les herbivores et 39 pour les granivores. A contrario, la part relative plus faible des charges variables dans les élevages d'herbivores est synonyme de quasi-fixités importantes, dont témoigne la superficie moyenne. Quant aux élevages de granivores, leur position reste intermédiaire.

En second lieu, on peut remarquer la plus grande homogénéité des résultats des producteurs de porcs, à l'opposé du groupe herbivores. Cette moindre dispersion se marque à travers l'écart-type peu important et au fait que le score minimum d'efficacité soit seulement de 0,67 pour les premiers contre 0,19 pour les seconds. Il faut noter que les élevages de granivores ont l'efficacité technique minimale la plus faible $(0,16)$, leur dispersion étant comprise entre celle des deux autres types d'élevages.

L'homogénéité des résultats obtenus par les producteurs de porcs par rapport aux autres productions ne signifie pas que les élevages possédant la plus petite SAU ou très spécialisés sont efficaces. En effet, l'approche DEA menée ici compare des techniques de production similaires. Ainsi, sont déclarées techniquement efficaces de très petites exploitations mais aussi de très grandes. De même, parmi les efficaces, nous trouvons des élevages spécialisés mais aussi des productions diversifiées. Les autres exploitations, dites « inefficaces ", sont comparées aux exploitations efficaces ayant la technique de production la plus proche pour déterminer leur score.

Afin de mettre en évidence quelques facteurs susceptibles d'expliquer les différences de score, les principales caractéristiques des élevages par classe d'efficacité sont présentées dans le tableau 3 . Il apparaît que les éleveurs de porcs efficaces sont plus jeunes et mieux formés que les éleveurs des autres groupes. Par ailleurs ils obtiennent un produit brut supérieur en utilisant moins de travail. La disponibilité de terre ne joue pas de rôle, les chiffres moyens de SAU étant très proches selon les catégories. En ce qui concerne les producteurs d'herbivores les choses sont plus complexes dans la mesure où ni l'âge ni la formation des éleveurs, ni la taille des exploitations ou la quantité de travail ne fournissent d'explication univoque. En fait, une étude plus détaillée montre que les élevages appartenant à la classe d'efficacité intermédiaire relève plutôt d'une orientation viande tandis que les élevages techniquement efficaces et inefficaces sont de type laitier. Les différences entre ces derniers tiennent à une meilleure utilisation des consommations intermédiaires chez les plus efficaces. Quant au groupe des granivores, il se rapproche de la situation des éleveurs de porcs avec un effet capital humain, les plus efficaces étant plus jeunes avec un niveau de formation agricole et général légèrement supérieur.

Sur un plan environnemental, on peut se demander si, étant donné la législation mise en œuvre en matière d'épandage, les niveaux d'azote produits par les éleveurs les plus efficaces sont les plus faibles. Ces résultats sont toutefois à prendre avec réserve puisque dans le RICA, seules les SAU sont renseignées. L'absence d'information sur les surfaces d'épandage hors exploitation introduit forcément un biais dans les mesures proposées dans le tableau 4. Ainsi, les exploitations spécialisées dans la production de porcs produisent plus d'azote lorsqu'elles sont déclarées efficaces sur un plan technique. Pour les deux autres échantillons considérés, la classe d'efficacité la plus faible (inférieure à 0,85 ) produit

\section{L'efficacité moyenne des élevages les moins performants $(<0,85)$ est très variable selon le groupe : de 0,48 pour les élevages avec herbivores à 0,78 pour les élevages de porcs seuls.}


Tableau 3. Caractéristiques des élevages selon leur niveau d'efficacité.

\begin{tabular}{|c|c|c|c|}
\hline & Porcs seuls & Herbivores & Granivores \\
\hline $\begin{array}{l}\text { Score d'efficacité de } \mathbf{1} \\
\text { Nombre d'exploitations } \\
\text { Superficie moyenne (ha) } \\
\text { Produit brut (F) } \\
\text { Travail (UTA) } \\
\text { Age moyen du chef d'exploitation } \\
\text { Formation agricole (\%) } \\
\text { - aucune } \\
\text { - primaire } \\
\text { - secondaire et supérieure } \\
\text { Formation générale (\%) } \\
\text { - aucune } \\
\text { - primaire } \\
\text { - secondaire }\end{array}$ & $\begin{array}{c}55 \\
27,7 \\
1819905 \\
1,75 \\
39,9 \\
\\
12,7 \\
21,8 \\
65,5\end{array}$ & $\begin{array}{c}76 \\
48,1 \\
355754 \\
1,77 \\
46,1 \\
\\
26,3 \\
48,7 \\
25,0\end{array}$ & $\begin{array}{c}71 \\
36,8 \\
858530 \\
1,89 \\
43,5 \\
\\
21,1 \\
38,2 \\
40,7\end{array}$ \\
\hline $\begin{array}{l}\text { Score d'efficacité compris entr } \\
\text { Nombre d'exploitations } \\
\text { Superficie moyenne (ha) } \\
\text { Produit brut (F) } \\
\text { Travail (UTA) } \\
\text { Age moyen du chef d'exploitation } \\
\text { Formation agricole (\%) } \\
\text { - aucune } \\
\text { - primaire } \\
\text { - secondaire et supérieure } \\
\text { Formation générale (\%) } \\
\text { - aucune } \\
\text { - primaire } \\
\text { - secondaire }\end{array}$ & $\begin{array}{c}85 \text { et } 1 \\
34 \\
29,6 \\
1706586 \\
1,92 \\
42,2\end{array}$ & $\begin{array}{c}13 \\
39,9 \\
186102 \\
1,79 \\
42,3\end{array}$ & $\begin{array}{l}29,4 \\
29,4 \\
41,2 \\
\\
\\
17,6 \\
35,3 \\
47,1\end{array}$ \\
\hline $\begin{array}{l}\text { Score d'efficacité inférieur à } \mathbf{0} \text {, } \\
\text { Nombre d'exploitations } \\
\text { Superficie moyenne (ha) } \\
\text { Produit brut (F) } \\
\text { Travail (UTA) } \\
\text { Age moyen du chef d'exploitation } \\
\text { Formation agricole (\%) } \\
\text { - aucune } \\
\text { - primaire } \\
\text { - secondaire et supérieure } \\
\text { Formation générale (\%) } \\
\text { - aucune } \\
\text { - primaire } \\
\text { - secondaire }\end{array}$ & $\begin{array}{c}18 \\
26,9 \\
1418588 \\
1,87 \\
46,8\end{array}$ & $\begin{array}{c}40 \\
49,3 \\
265802 \\
1,76 \\
44,4\end{array}$ & $\begin{array}{c}47 \\
39,5 \\
755982 \\
2,14 \\
46,6 \\
\\
19,1 \\
31,9 \\
48,9\end{array}$ \\
\hline
\end{tabular}

Tableau 4. Niveau d'azote des élevages selon leur niveau d'efficacité.

\begin{tabular}{|l|c|c|c|}
\hline & Porcs seuls & Herbivores & Granivores \\
\hline Score d'efficacité de 1 & & & \\
Nombre d'exploitations & 55 & 76 & 71 \\
Azote organique (kg/ha) & 824 & 85 & 172 \\
Azote minéral (kg/ha) & 127 & 178 & 130 \\
\hline \multicolumn{2}{|l|}{ Score d'efficacité compris entre $\mathbf{0 , 8 5}$ et 1 } & & \\
Nombre d'exploitations & 34 & 13 & 17 \\
Azote organique (kg/ha) & 240 & 70 & 118 \\
Azote minéral (kg/ha) & 132 & 144 & 144 \\
\hline Score d'efficacité inférieur à $\mathbf{0 , 8 5}$ & & & 47 \\
Nombre d'exploitations & 18 & 40 & 163 \\
Azote organique (kg/ha) & 208 & 77 & 187 \\
Azote minéral (kg/ha) & 128 & 205 & \\
\hline
\end{tabular}

moins d'azote organique que la classe la plus efficace (score de 1) mais utilise plus d'engrais. On constate alors qu'une amélioration de l'efficacité technique des producteurs d'herbivores et de granivores peut conduire à une réduction du niveau d'azote tout en augmentant le produit brut et donc le revenu des éleveurs. Cette compatibilité entre amélioration de l'efficacité technique et réduction des rejets azotés n'est pas généralisable aux producteurs de porcs seuls.

D'autre part, un score d'efficacité moyen de 0,94 chez les producteurs de porcs signifie une diminution moyenne possible de $6 \%$ de tous les facteurs variables et du niveau d'azote organique pour les éleveurs présentant une inefficacité technique. Cette réduction potentielle est de $16 \%$ chez les éleveurs d'herbivores et de $13 \%$ chez les éleveurs de granivores. En termes d'ajustement des charges variables, ces diminutions permettraient, si elles sont réalisées, une réduction moyenne du coût variable de $71604 \mathrm{~F}$ pour le groupe des porcs purs, $53063 \mathrm{~F}$ pour celui des herbivores et $96807 \mathrm{~F}$ pour celui des granivores. Cependant ces ajustements ne sont pas effectifs pour l'ensemble des producteurs de chaque échantillon. En effet, comme nous l'avons vu dans le tableau 3 , pour les producteurs dits efficaces (score de 1), aucune diminution des facteurs ou de l'azote organique n'est nécessaire pour améliorer leur situation. Par contre, pour ceux dont le score est inférieur à 0,85 , les ajustements seront beaucoup plus importants que pour les éleveurs ayant un score compris entre 0,85 et 1 . En effet, les réductions potentielles sont comprises entre 2 et $7 \%$ pour les élevages appartenant à la classe intermédiaire des scores et entre 22 et $52 \%$ pour ceux ayant le niveau d'efficacité le plus faible.

Pour déterminer les secteurs sur lesquels les producteurs disposent le plus de marges de manœuvre pour améliorer leur efficacité, le programme DEA fournit une information sur les facteurs de production devant être ajustés en priorité en identifiant les intrants "surutilisés » par les exploitations présentant une inefficacité technique par rapport à d'autres élevages ayant la même technique de production mais déclarés efficaces. Ainsi, en moyenne sur l'échantillon sans modifier le niveau de production animale, les producteurs d'herbivores peuvent améliorer leur efficacité en réduisant dans un très court terme leurs charges variables de l'ordre de $19 \%$ dont $20 \%$ pour les engrais et les charges pour les cultures et $16 \%$ pour les charges liées à l'activité d'élevage. Dans un plus long terme, des ajustements de certains facteurs fixes sont aussi possibles qui concernent le matériel et les bâtiments. Pour les éleveurs de granivores, on observe une réduction potentielle des facteurs variables de $21 \%$ avec une baisse possible de $30 \%$ pour les achats d'engrais et de $23 \%$ pour les charges associées à l'activité de culture. L'ajustement sur les facteurs fixes concerne la terre et le matériel (16\%). Enfin, pour les producteurs de porcs, les marges de manœuvre 
sont plus faibles, étant donné le niveau de spécialisation. Toutefois on note une possibilité de baisse des facteurs variables en moyenne de $14 \%$ dont $9 \%$ pour les engrais et $15 \%$ pour les charges pour les cultures. Comme nous l'avions déjà observé dans le tableau 3, une amélioration de l'efficacité technique se traduit à plus long terme par une réduction potentielle de $16 \%$ de la terre.

En ce qui concerne le niveau d'azote organique, une amélioration de l'efficacité technique a pour conséquence une augmentation de $2 \%$ de celui-ci chez les éleveurs de porcs seuls et une réduction de respectivement 8 et $5 \%$ chez les producteurs d'herbivores et de granivores.

Ainsi, on constate que quel que soit le type d'élevage, l'ajustement le plus important à court terme s'effectue sur les charges liées à la production de végétaux, alors qu'à long terme, il concerne la terre pour les exploitations produisant des porcs et des granivores et sur le matériel pour celles des groupes herbivores et granivores.

\section{4 / Mesure de l'efficacité d'échelle}

Des mesures d'efficacité d'échelle ont été calculées pour les trois échantillons de notre étude (tableau 5). L'efficacité d'échelle est en moyenne plus élevée chez les producteurs de porcs purs $(0,97)$ et chez les granivores $(0,96)$. que chez les herbivores $(0,90)$. Ainsi, près de 3 $\%$ de l'inefficacité s'explique par l'échelle de production mise en œuvre par les producteurs de porcs seuls. En effet, près de $45 \%$ des exploitations sont dans une situation d'équilibre concurrentiel de long terme alors que $20,6 \%$ d'entre elles peuvent augmenter leur niveau de production de manière plus que proportionnelle à l'augmentation des facteurs de production car les rendements d'échelle sont croissants. De plus, 34,6 \% des élevages ont une taille de production où les rende- ments d'échelle sont décroissants, c'est-à-dire que toute augmentation des intrants conduit à une hausse proportionnellement plus faible du niveau des produits.

Les résultats obtenus pour les élevages de granivores sont très proches de ceux observés pour ceux produisant des porcs purs. Par contre, l'efficacité d'échelle plus faible de l'orientation herbivores se traduit aussi par un nombre plus important d'exploitations en situation de rendements d'échelle constants et croissants que dans les deux autres groupes.

Lorsqu'on compare les informations entre classes d'efficacité, on constate que les éleveurs les plus efficaces techniquement (score de 1) sont à plus de $70 \%$ en situation de rendements d'échelle constants. Par contre, pour la classe intermédiaire d'efficacité, on trouve la plus grande proportion de rendements décroissants chez les producteurs de porcs $(62$ $\%$ ) suivis de ceux produisant des granivores $(41 \%)$ et des herbivores $(15,5 \%)$. Ces derniers sont plutôt dans une situation de rendements d'échelle constants (46\%) ou croissants (38 $\%)$. Les producteurs de granivores présentent quant à eux des rendements d'échelle croissants $(53 \%)$ et décroissants $(41 \%)$. Enfin, les éleveurs de porcs et d'herbivores les moins efficaces sont dans une situation de rendements d'échelle croissants et décroissants alors que $66 \%$ de ceux produisant des granivores présentent des rendements d'échelle décroissants.

\section{Conclusion}

Ce travail à caractère exploratoire met en évidence d'une part l'intérêt de la notion d'inefficacité pour mieux appréhender les performances des élevages et d'autre part le caractère opérationnel de l'approche non paramétrique. Sur une base plus restreinte, tant en nombre d'exploitations qu'en type

Tableau 5. Efficacité d'échelle et rendements d'échelle.

\begin{tabular}{|c|c|c|c|c|}
\hline \multirow{2}{*}{ Efficacité technique } & \multirow{2}{*}{ Efficacité d'échelle } & \multicolumn{3}{|c|}{ Rendements d'échelle (\% d'exploitations) } \\
\hline & & constants & croissants & décroissants \\
\hline $\begin{array}{l}\text { Porcs seuls } \\
\text { - de } 1 \\
\text { - comprise entre } 0,85 \text { et } 1 \\
\text { - inférieure à } 1 \\
\text { Moyenne }\end{array}$ & $\begin{array}{l}0,98 \\
0,94 \\
0,99 \\
\mathbf{0 , 9 7}\end{array}$ & $\begin{array}{l}76,4 \\
11,8 \\
11,1 \\
\mathbf{4 4 , 8}\end{array}$ & $\begin{array}{l}12,7 \\
26,5 \\
33,3 \\
\mathbf{2 0 , 6}\end{array}$ & $\begin{array}{l}10,9 \\
61,8 \\
55,6 \\
\mathbf{3 4 , 6}\end{array}$ \\
\hline $\begin{array}{l}\text { Herbivores } \\
\text { - de } 1 \\
\text { - comprise entre } 0,85 \text { et } 1 \\
\text { - inférieure à } 1 \\
\text { Moyenne }\end{array}$ & $\begin{array}{l}0,91 \\
0,97 \\
0,85 \\
\mathbf{0 , 9 0}\end{array}$ & $\begin{array}{r}72,4 \\
46,2 \\
2,5 \\
\mathbf{4 8 , 1}\end{array}$ & $\begin{array}{l}13,2 \\
38,5 \\
50,0 \\
\mathbf{2 7 , 1}\end{array}$ & $\begin{array}{l}14,5 \\
15,5 \\
47,5 \\
\mathbf{2 4 , 8}\end{array}$ \\
\hline $\begin{array}{l}\text { Granivores } \\
\text { - de } 1 \\
\text { - comprise entre } 0,85 \text { et } 1 \\
\text { - inférieure à } 1 \\
\text { Moyenne }\end{array}$ & $\begin{array}{l}0,97 \\
0,95 \\
0,96 \\
\mathbf{0 , 9 6}\end{array}$ & $\begin{array}{r}76,1 \\
5,9 \\
8,5 \\
43,7\end{array}$ & $\begin{array}{r}9,9 \\
52,9 \\
25,5 \\
\mathbf{2 0 , 7}\end{array}$ & $\begin{array}{l}14,1 \\
41,2 \\
66,0 \\
\mathbf{3 5 , 6}\end{array}$ \\
\hline
\end{tabular}

Cette méthode met en évidence les ajustements à réaliser dans les élevages de moindre efficacité, par exemple, dans cette étude, la réduction des charges liées à la production végétale. 
d'orientation, Boussemart et Dervaux (1994) ont montré la cohérence du diagnostic d'efficacité productive avec des indicateurs techniques, comptables et financiers d'un groupe de naisseurs-engraisseurs. Ici, par l'analyse de trois échantillons de taille convenable nous arrivons à caractériser des systèmes de production les uns par rapport aux autres, mais aussi des sous-groupes à l'intérieur de ces systèmes.

Parmi les résultats les plus significatifs, on notera l'importance d'une bonne caractérisation des technologies de production. En effet, en présence de systèmes où les facteurs variables jouent un rôle prépondérant, la plus grande homogénéité de la technologie permet une meilleure caractérisation des ajustements possibles pouvant être effectués à court terme. Pour les systèmes de production où la combinaison factorielle repose surtout sur le foncier ou le travail familial, des améliorations sont possibles mais obligatoirement à plus long terme.

Dans une perspective opérationnelle, nous avons raisonné en comparant les caractéristiques factorielles et humaines des exploitations peu ou moyennement efficaces, à celles des plus efficaces techniquement. Cette approche s'apparente dans son esprit aux méthodes classiques de gestion tête-intermé- diaire-queue dont l'intérêt n'est pas remis en cause. Ces techniques donnent toujours des résultats intéressants (cf. Bébin et al 1995). Toutefois la DEA, en chiffrant par type de facteurs les possibilités de gain d'efficacité qui s'ouvrent aux éleveurs, permet d'aller plus loin. En mettant l'accent sur les facteurs faisant l'objet d'une sur-utilisation en comparaison des combinaisons factorielles mises en œuvre par les exploitations les plus efficaces, la DEA fournit un levier d'action aux agents du développement. Au-delà, la possibilité d'intervenir au cas par cas est ouverte. En effet, la méthode permet de caractériser chaque individu, mettant en évidence les possibilités de résorption des inefficacités qui lui sont propres. L'aide au diagnostic, puis au conseil est ainsi grandement améliorée.

Enfin, notons l'aspect relatif de la notion d'efficacité puisque l'on compare des individus entre eux. Dès que l'on élargit la population de référence, le risque est alors de faire entrer dans l'échantillon des exploitations encore plus efficaces que les précédentes. Par ailleurs, rien n'empêche l'élaboration de frontières de production théoriques prenant en compte un certain nombre d'innovations à caractère encore expérimental. Ainsi pourraiton apprécier une sorte d'inefficacité " absolue » au lieu d'une inefficacité relative.

\title{
Références bibliographiques
}

Bébin D., Lherm M., Liénard G., 1995. Quels résultats techniques et économiques en grands troupeaux de vaches allaitantes ? Le cas du Charolais. INRA Prod. Anim., 8, 213-225.

Boussemart J.P., Dervaux B., 1994. Diagnostic de l'efficacité productive par la méthode DEA. Application à des élevages porcins. Cahiers d'Economie et Sociologie Rurales, 31, 43-58.

Chambers R.G., 1988. Applied production analysis. A dual approach. Cambridge University Press, 331 p.

Charnes A., Cooper W.W., Rhodes E., 1978. Measuring the efficiency of decision making units. Eur. J. Operations Res., 2, 139-156.

Debreu G., 1951. The coefficient of ressource utilization. Econometrica, 19, 273-292.
Farrell M.J., 1957. The measurement of productive efficiency. J. Royal Statistical Soc., Series A 120 part. 3, 253-290.

Glais M., 1990. Gestion et Economie. In : Greffe, Mairesse, Reiffers (eds), Encyclopédie économique, tome 1, 215-251. Ed. Economica, Paris.

Lassègue P., 1981. Qu'est-ce que la gestion ? Tout. Qu'a-t-elle été jusqu'à présent ? Rien. Que veut-elle devenir ? Quelque chose. Mélanges offerts à PDVigreux. IPA-IAE Toulouse.

Mairesse J., 1993. Economie agricole : les raisons d'une prééminence. Cahiers d'Economie et Sociologie Rurales, 29, 95-99.

Piot-Lepetit I., 1995. Les excédents d'intrants polluants dus à des inefficacités des producteurs agricoles. Thèse de doctorat en Sciences Economiques de l'Université de Bordeaux I, 297 p.

\begin{abstract}
Breathing room in farm management as determined by inefficiency measurements.

This paper begins with a brief survey of the literature relating to the idea of inefficiency and how it relates to management. The concept of technical inefficiency is defined in this paper as being the difference between the observed production level and the maximum attainable level of output

which could be theoretically reached if the input were to be used in a technically efficient manner. Measure of technical efficiency of three different farm types (pigs alone, pigs and herbivores, pigs and granivores (poultry)) is given for the year 1991 using a nonparametric method, Data Envelopment Analysis (DEA) based on linear programming techniques.
\end{abstract}


The initial results, on average, revealed that the producers of pigs alone had the greatest efficiency. These farmers tended to be younger and had received better training than the two other groups of farmers. These results also seemed to be linked to the greater homogeneity in production techniques in use and to the preponderence of variable factors involved in the production process as compared to the quasi-fixed factors. For the pig and herbivore farmers, both the most and least efficient producers tended to involve dairy farms. The performance differences between these were explained by a better use of intermediate level consumptions on the more efficient farms. The pig and poultry group had efficiency values approaching those of the pig alone group, but their characteristics were less pronounced. Environmentally, the possibility of increasing technical efficiency and while decreasing the production of nitrogen waste seemed only possible on the mixed farms (pig and herbivore or granivore). The maneuvering room available for improving farm technical efficiency concerns, in the short term, the costs relating to the production of vegetable products, primarily, and in the long term, the cost of land and material, in particular for farms involving bovines. In conclusion, the efficiency measure indicated that cost savings are possible if the production scale is modified on 52 $\%$ of the farms considered, no matter what their type.

PIOT-LEPETIT I., RAINELLI P., 1996. Détermination des marges de manœuvre des élevages à partir de la mesure des inefficacités. INRA Prod. Anim., 9 (5), 367-377. 